Culture et histoire dans l'espace roman

4 | 2010

Les représentations du corps dans la litterature latinoaméricaine

\title{
Le corps chez Chico Buarque : une question d'« extimité »
}

\section{Ana Maria Clark Peres}

\section{(2) OpenEdition}

\section{Journals}

Édition électronique

URL : https://journals.openedition.org/cher/8259

DOI : $10.4000 /$ cher.8259

ISSN : 2803-5992

Éditeur

Presses universitaires de Strasbourg

Édition imprimée

Date de publication : 30 juin 2010

Pagination : 149-158

ISBN : 978-2-35410-007-0

ISSN : 1968-035X

Référence électronique

Ana Maria Clark Peres, «Le corps chez Chico Buarque : une question d' « extimité » », reCHERches [En ligne], 4 | 2010, mis en ligne le 15 décembre 2021, consulté le 26 janvier 2022. URL : http:// journals.openedition.org/cher/8259 ; DOI : https://doi.org/10.4000/cher.8259

\section{cc) (†) (2)}

Ce(tte) œuvre est mise à disposition selon les termes de la Licence Creative Commons Attribution Pas d'Utilisation Commerciale - Partage dans les Mêmes Conditions 4.0 International. 


\title{
Le corps chez Chico Buarque: une question d' "extimité »
}

\author{
Ana Maria Clark Peres \\ Université fédérale de Minas Gerais
}

\section{Le corps dans les chansons de Chico Buarque}

Les références au corps traversent l'œuvre de Chico Buarque1. Dans les paroles de plusieurs de ses chansons, Chico (comme nous le connaissons au Brésil) met en scène le corps de l'homme ou de la femme, sous des lumières variées.

Dans les années 60, par exemple, lorsqu'il enregistre ses premiers disques, il cherche à incarner de préférence l'homme ordinaire, comme le sambiste du morne, qui chante l'amour de façon ingénue: "Je veux chanter l'amour / Avant que l'amour finisse $»^{2}$ ou «Je fais de la samba et l'amour la nuit entière/ Je n'ai de comptes à rendre à personne $»^{3}$.

En ce qui concerne le corps, il est déjà annoncé que la chanson est capable de porter sur scène le corps de l'aimée, toujours associée à l'univers de la samba et du carnaval, comme dans «Demain on ne sait pas»: «Je veux voir

1 Ou mieux, de Francisco Buarque de Hollanda, compositeur et interprète consacré de la musique populaire brésilienne, en plus d'être l'auteur de pièces de théâtre et de romans traduits en français et dans diverses autres langues. Il faut remarquer que, né à Rio de Janeiro, en juin 1944, il est fils de l'historien Sérgio Buarque de Hollanda, un des intellectuels brésiliens les plus importants, auteur, entre autres œuvres, de Raízes do Brasil [Les racines du Brésil], qui aborde des aspects cruciaux de l'histoire de la culture brésilienne. (Buarque de Hollanda 1973).

2 «Demain on ne sait pas».

3 «Samba et amour». 
la samba chauffer/ Dans le corps de la femme porte-étendard/ Que ma guitare va lever».

Dans les années 70, apogée de la dictature militaire qui a sévi dans notre pays de 1964 à 1985, les chansons de Chico changent de ton: les paroles parlent maintenant d'un corps torturé, exilé («Aux quatre coins ton corps / Brisé / Banni») dont la souffrance devient évidente: «Aux quatre coins tes hurlements / Ton cri/ Horrible $»^{4}$. Ou encore: «Si ton corps te fait mal/ Dis que oui/ Ils le tordent un peu plus / Dis que oui/ S'ils te donnent un coup / Dis que oui $»^{5}$. En hommage à la styliste brésilienne Zuzu Angel qui, à l'époque, cherchait inlassablement des informations sur la mort de son fils, Stuart Angel, prisonnier politique qui mourut mystérieusement en prison, le compositeur devait écrire les vers suivants: "Qui est cette femme/ Qui chante toujours la même chose/ Je voulais seulement couvrir mon ange/ Et laisser reposer son corps ${ }^{6}$.

En plus de dénoncer ce corps torturé, Chico commence à incorporer, dans les paroles de ses chansons, la voix féminine, donnant libre cours à l'érotisme: "Quand tu me regardas bien dans les yeux / Et ton regard était d'adieu/ Je jure que je ne l'ai pas cru/ Je t'ai trouvé étrange/ Je me suis penchée / Sur ton corps et j'ai douté/ Je me suis traînée et je t’ai griffé/ Je me suis accrochée à tes cheveux/ à tes poils [...]/ Pour te montrer que je suis encore à toi $»^{7}$. Ou encore: "Il connaît les chemins/ De cette terre à moi / Dans mon corps il se cacha/ Mes forêts il traversa/Sur mes fleuves / Dans mes bras / [...] Dans les entrailles tant de aïe ${ }^{8}$. Encore un exemple: "Je veux rester sur ton corps fait tatouage/ [...] Je veux jouer en toi fait danseuse / [...] Je veux être la cicatrice riante et corrosive / Marquée au froid, au fer et au feu / De chair vive $»^{9}$. Voyons un nouvel exemple de cet érotisme: «Oh mon amour/ Il a une façon douce qui n'est qu'à lui/ De faire le tour de mon corps / De me baiser la poitrine / Me baiser le ventre / Et me laisser de braise / Il jouit de mon corps / Comme si mon corps était son chez lui [...]/ Mon corps est le témoin/ Du bien qu'il me fait ${ }^{10}$. Ici, c'est la prostituée

\footnotetext{
4 «Serpent de verre».

5 «Sort vainqueur dans la vie qui dit oui».

6 «Angélica».

7 «Derrière la porte».

8 «Tais-toi, Barbara».

9 «Tatouage».

10 «Mon amour».
} 
qui dit: «N'importe quel amour/ Me satisfait/ N'importe quelle chaleur/ N'importe quel jeune homme ${ }^{11}$.

Notons, par conséquent, que Chico se réfère désormais non seulement au corps, mais qu'en plus il «prête» son propre corps afin de permettre à la voix féminine de se manifester. Au début de sa carrière, d'ailleurs, il avait déjà «emprunté» le corps pour la manifestation de la voix du sambiste populaire, comme nous l'avons vu. Et il le fit de façon très érudite, imprégné de ses lectures d'auteurs étrangers ${ }^{12}$.

Le compositeur et cinéaste Ruy Guerra va jusqu'à affirmer que Chico est le «cheval» des «sambistes" et «mères-maquerelles», dans une allusion au candomblé, dans lequel le «fils de saint» (le «cheval») «prête» aussi son corps pour que les Orixás se manifestent ${ }^{13}$.

Toujours sur le candomblé, voyons ce que nous dit René Bastide, considéré comme le plus grand spécialiste des religions africaines au Brésil: «les dieux africains [les Orixás] ne peuvent vivre que dans la mesure où ils se réincarnent dans la chair de leurs fidèles. Et c'est pourquoi le centre du culte public est la crise de la possession» (Bastide 200: 44). Chacun des cultes est dédié à une divinité, mais on peut remarquer les moments qui suivent, comuns à tous: le sacrifice d'un animal, qui est passé, ensuite, les mains de la cuisinière qui va préparer les aliments des dieux; le padé de Exú14; l'appel musical (jeu musical des tambours); les danses et cantiques: «le rythme de plus en plus rapide, de plus en plus implorant, finit par ouvrir les muscles, les viscères, les têtes à la pénétration du dieu si longtemps attendu » (Bastide 2000 : 50). Se produit alors la "crise de possession», qui peut s'avérer plus ou moins violente. De même qu'arrive la «danse des dieux»:

11 «N'importe quel amour».

12 Voyons un exemple de cette érudition, dans une entrevue avec le journaliste brésilien Hamilton Ribeiro: «Parmi les livres de mon père [j’ai lu] la collection de la Pléiade. Et je trouvais génial le fait de lire en français. J'ai lu quasiment tous les classiques français et la traduction française des russes. Je suis arrivé à Céline, me croyant le découvreur de Voyage au bout de la nuit. Là, un collègue m'a dit que je n'avais même pas un soupçon de formation [littéraire] brésilienne. J'ai plongé dans Macunaíma, Graciliano, Guimarães Rosa, Drummond, Bandeira». (Ribeiro 1972). http://www.chicobuarque.com.br/texto/ index.html

13 «Le mot candomblé désigne à Salvador de Bahia [...] à la fois le lieu et les fêtes rituelles d'origine africaine qu'on y célèbre régulièrement. Ce territoire - ce terreiro - n'est pas seulement une étendue sacrée parmi d'autres, mais le socle invisible d'une intense activité physique et mentale qui associe tous les sens du corps [...]» (Bastide 2000: 13-14).

14 «Exú, c'est le diable; il pourrait troubler la cérémonie si on ne lui rendait pas hommage» (Bastide 2000: 47). 
[...] les gestes ont une autre beauté, les pas de danse atteignent une étrange poésie. Ce ne sont plus de petites couturières à la journée, des cuisinières ou des laveuses qui tournent au son des tambours dans les nuits de Bahia, c'est Omolú recouvert de paille, c'est Xangô rouge et blanc, c'est Yemanjá peignant ses cheveux de varech. Les visages sont devenus masques, se sont métamorphosés; ils ont perdu les rides du travail quotidien, les stigmates de la vie de tous les jours, avec ses soucis et ses misères [...] (Bastide 2000 : 52-53).

Finalement, après de nouveaux cantiques, qui visent à renvoyer les Orixás, «les paupières closes s'ouvrent, le visage perd son masque divin, la personnalité normale réapparaît». Et il y a un "repas communiel» (Bastide 2000: 53). Bastide nous éclaire encore sur la possession: "Ce que nous désignons par phénomène de possession pourrait donc mieux se définir comme phénomène de transformation de personnalité» (Bastide 2000: 220).

Mais retournons à Chico Buarque. Bien que, comme «cheval », il "prête» son corps pour que la réalité de certaines femmes et de l'homme ordinaire apparaisse dans les paroles de ses chansons, il faut noter que, chantant ces chansons, il ne se transforme pas, ne se métamorphose pas, comme cela arrive dans le candomblé avec les «chevaux des dieux». Par conséquent, aussi motivante (et valide) que soit l'association proposée par Ruy Guerra, je crois que l'on peut avancer d'un pas en pensant le lieu du corps dans la création de Chico, ce que nous verrons plus loin.

D'ailleurs, dans son processus de création, un renouvellement important va se manifester à partir des années 90: Chico devient romancier, avec la publication d'Embrouille, en portugais pour la première édition, en 1991, sous le titre de Estorvo; de Court-circuit, publié au Brésil en 1995, sous le titre de Benjamim, et de Budapest, lancé au Brésil en 2003.

\section{Les couples d'opposition chez Chico Buarque : où loger le corps?}

Avant de nous arrêter sur le roman Budapest, il serait intéressant de souligner certaines lectures de l'œuvre de Chico qui vont y déceler des couples d'(apparente) opposition.

Parmi plusieurs couples relevés, j'aimerais n'en retenir que quatre principaux, qui sont: national/ étranger; privé/ publique; populaire/ érudit; masculin/ féminin.

En ce qui concerne le premier couple (national / étranger), le compositeur déclare dans sa chanson "Pour tous», de 1993, dans une espèce de synthèse 
de son parcours jusqu'alors: «Je fais la route depuis longtemps/ Je suis un artiste brésilien ». D’ailleurs, on l'a déjà jugé au Brésil « unanimité nationale » et élu le musicien brésilien du $\mathrm{XX}^{\mathrm{e}}$ siècle. Mais, comme nous l'avons vu avant, curieusement, la formation littéraire de ce Brésilien si «typique» est nettement étrangère.

À propos du couple privé/ public, Eneida Maria de Souza affirme:

Chico cultive le rêve de se transformer en artiste invisible, ne cédant en rien à la sollicitation schizophrénique des médias. Le compositeur se cache sous la peau de l'écrivain, l'artiste déteste la scène et le spectacle, atteint la popularité pour se montrer contraire à elle et se consacrer beaucoup plus par la négation de la célébrité. (Souza 2006: 56)

Reprenant le couple national/ étranger, et comme en dédoublement, nous avons le couple populaire / érudit, une fois que le national, chez Chico Buarque, est très souvent entendu comme «national-populaire »; l'érudition, quant à elle, est étrangère pour Chico.

Finalement, il faudrait rappeler que l'un des couples les plus évoqués dans l'œuvre de Chico est celui du masculin/féminin, ce à quoi nous avons fait allusion au début de ce texte, quand nous avons proposé des exemples du corps féminin chanté ou incarné par le compositeur.

Comme l'indique Adélia Bezerra de Menezes:

D'un côté, sa production offre une vision très masculine du féminin, dans un lyrisme profondément corporel. [...] D'un autre côté, la chanson de Chico Buarque, d'innombrables fois, présente un je lyrique féminin (l'anima de l'Auteur qui affleure, diraient les junguiens). De fait, le poète est cet êtrelà à qui est donné, plus qu'à d'autres, le pouvoir de manifester la vie des affects. [...] C'est ainsi que dans les chansons de Chico Buarque émerge la parole de la femme, sous une perspective, parfois, incroyablement féminine. (Menezes 2001: 15-20).

Lorsque nous associons ces couples d'opposition à la question du corps, thème de ce travail, la première question qui vient est la suivante: où situer le propre corps du compositeur-écrivain? Ce corps serait-il parfaitement ajusté aux limites de son pays (Chico est-il, de fait, le "Brésilien type»?) ou s'installerait-il de façon commode, parfois, dans des cultures étrangères? S'agirait-il d'un corps reclus ou d'un corps qui s'offre à la lumière des projecteurs? Chico se présenterait-il comme ayant un corps «commun», qui partage sa vie, sa routine, comme tant d'autres corps semblables à lui, ou un corps qui se distingue par l'érudition et par le travail intellectuel? S'agit-il d'un corps masculin qui incarne un corps féminin, parfaitement assimilable, 
ou qui considère le corps de la femme comme à part? En résumé: en plus de se référer au corps, d'emprunter son corps pour faire passer d'autres voix, quel lieu Chico Buarque occuperait-il dans la culture contemporaine? Et plus encore: où se situe le corps qu'il chante, qu'il écrit? Est-il possible de le placer de façon définitive sur l'un ou l'autre point, qui s'excluent mutuellement?

Afin de développer ces questions, retournons aux couples d'opposition rencontrés dans l'œuvre de Chico Buarque. Après les avoir réduits à quatre principaux, nous chercherons maintenant à les réduire à un couple paradigmatique, le dedans/dehors. En l'abordant superficiellement, il ne nous paraît pas difficile d'établir quel est le dedans et quel est le dehors: le national, le privé et le populaire se réfèrent au dedans, tandis que l'étranger, le publique et l'érudit se réfèrent au dehors. Mais l'étranger ne serait-il pas pour Chico, parfois, ce qui est plus intime, se mettant en rapport, donc, avec le dedans? Nous ne pouvons oublier, répétons-le, la grande familiarité, la grande intimité de Chico avec les textes étrangers, ce qui atteste, sous d'autres aspects, sa grande érudition. Et encore: pour reprendre la position d'Eneida Maria de Souza, dans l'essai déjà cité, nous dirons que l'aversion du compositeur-écrivain au fait de s'exposer publiquement ou, en d'autres termes, sa recherche infatigable du privé, est un des facteurs qui font de lui une figure si connue. À tant vouloir se maintenir dedans, échapperait-il dehors?

Mais, de façon plus marquée que dans d'autres couples, c'est peut-être dans le couple masculin-féminin que le dedans/dehors se confond le plus. Le féminin serait-il le «dedans», c'est-à-dire, l'anima de l'auteur, comme l'indique Adélia Menezes? Ou le «dehors» (une altérité radicale)? Voyons ce que nous dit Chico à ce propos dans une entrevue avec un journaliste brésilien: "Je pense que c'est une contradiction de dire que j'exprime très bien le sentiment féminin, parce que pour moi les femmes sont un énorme mystère» (Eichemberg 2006 ${ }^{15}$ ).

\section{Budapest et la notion d'“extimité»: un dedans qui est dehors ou un dehors qui est dedans?}

Prenant en compte l'hypothèse que je suis en train de développer dans un projet de recherche, selon laquelle le dernier roman de l'auteur, Budapest,

15 Voir http//www.chicobuarque.com.br/texto/index.html 
« recueille» et "épure» l'œuvre de Chico Buarque ${ }^{16}$, je vise à donner une nouvelle dimension, dans ce roman, à partir de l'une de ses lectures, au couple paradigmatique dedans/ dehors.

José Costa, narrateur-personnage de Budapest, vit à Rio de Janeiro, travaillant comme nègre: il écrit pour d'autres des monographies, des dissertations, des requêtes d'avocat et aussi, comme il l'affirme, «des lettres d'amour, d'adieu, de désespoir, des chantages, des menaces de suicide» (Buarque 2005: 20).

Soulignons que, une fois de plus, la question du corps et, pourquoi pas, le thème du "cheval» dans le candomblé y sont présents, une fois que le nègre prête son corps, mais de façon si radicale, cherchant à disparaître, à perdre son propre corps, à devenir un fantôme, comme l'évoque assez bien l'expression anglaise ghost writer.

Quant au narrateur de Budapest, il atteint le sommet de sa carrière en écrivant Le Gynographe, autobiographie érotique d'un cadre supérieur allemand qui, en arrivant au Brésil, apprend à écrire le portugais sur le corps des femmes (toujours le corps). Voyons comme José Costa entre dans la peau de l'Allemand:

Je prenais au hasard une des vingt cassettes que j'avais enregistrées, j'écoutais distraitement sa voix, je posais les doigts sur le clavier, et j'étais un homme blond au teint rose sept ans auparavant quand, après avoir embarqué à Hambourg, j'étais entré dans la baie de Guanabara. [...] au bout de trois mois de désarroi, j'ai senti que j'avais l'histoire de l'Allemand sur le bout des doigts. Je me suis mis à écrire spontanément, à un rythme qui n'était pas le mien [...] (Buarque 2005: 31-39).

$\mathrm{Au}$ retour d'un congrès d'écrivains anonymes en Turquie, le nègre se retrouve à Budapest «à cause d'une escale imprévue, alors qu'il volait d'Istanbul à Francfort, où il avait une correspondance pour Rio». Il y passe la nuit, où il se trouve en contact avec la langue hongroise, qui le fascine; comme il l'affirme, c'est «la seule langue du monde, disent les mauvaises langues, que le diable respecte» (Buarque 2005: 12). De retour à Rio de Janeiro, il finit par connaître, au consulat de Hongrie, l'«éminent poète hongrois Kocsis Ferenc», ce qui l'incite à retourner en Hongrie et lui fait prendre finalement cette décision. Entre en scène, alors, Zsoze Kósta, comme on va le nommer désormais à Budapest. Totalement investi à dominer parfaitement la langue hongroise, il commence à prendre des

16 Nous optons pour le mot «épurer» comme on l’utilise en minéralogie. 
cours avec Kriska, qui devient aussi sa petite amie. Chaque fois plus à l'aise avec le hongrois, le protagoniste finit par écrire des vers à la place de Kocsis Ferenc, lesquels apportent au poète la reconnaissance du public et de la critique, dont il ne profitait plus. Après le lancement du livre, et enivré par sa création, Kósta déclame pour Kriska quelques-uns des tercets qu'il a composés sous le nom du poète hongrois. Il lui demande ce qu'elle en pense, et voici la réponse (décevante) qu'elle lui fait: "On dirait qu'ils ne sont pas hongrois ces poèmes». Et elle ajoute: "c'est comme si c'était écrit [je souligne] avec un accent étranger [...]. Cette sentence, elle l'a émise presque en chantant, et c'est ce qui m'a mis hors de moi. J'ai pris mon assiette de spaghettis et je l'ai lancée sur le mur» (Buarque 2005: 126).

L'emportement de Kósta se doit, je crois, au fait d'être confronté à un trou, c'est-à-dire, à un dehors (un accent étranger) qui se présente dans le texte, au cœur même de ce texte, dans une relation d' «extimité» avec lui.

«Extimité» est un néologisme créé par Lacan dans Le Séminaire, Livre VII, L'éthique de la psychanalyse (1959-1960). Dans ce séminaire, se référant à la Chose, qu'il introduit à partir de das Ding, de Freud, Lacan parle d'un «lieu central», d'une "extériorité intime», c'est-à-dire, d'une " extimité» (Lacan 1986: 167) ${ }^{17}$. Quelques années plus tard, dans Le séminaire, Livre XVI, D’un Autre à l'autre (1968-1969), reprenant le séminaire précédent, Lacan se réfère encore à une centralité, à un champ de jouissance, à «ce qui nous est le plus prochain, tout en nous étant extérieur». Et il ajoute: «Il faudrait faire le mot extime pour désigner ce dont il s'agit» (Lacan 2006 : 224). C'est-à-dire qu'au plus intime, se loge un extérieur.

Selon le propos de Kriska, à l'intérieur du corps textuel (écrit) se trouve un objet étrange (un accent/ un ton de voix). Il nous semble important de remarquer que la notion d' «extimité» renverse l'opposition monde

17 Quant à das Ding, notons que, selon Lacan, il s'agit du premier extérieur, autour de quoi s'oriente le cheminement du sujet par rapport au monde de ses désirs. Dans «L'esquisse d'une psychologie scientifique», de 1895, Freud nous parle de la première expérience de satisfaction de l'enfant: le nouveau-né ressent un abandon initial, c'est-à-dire qu'il a faim et qu'il a besoin de l'aide d'autrui, normalement de la mère, qui fait cesser l'inconfort, et devient ainsi responsable de la première expérience de satisfaction de l'enfant. Cette première satisfaction laisse des traces dans l'appareil psychique, mais le plaisir ressenti à ce moment inaugural devrait ne plus pouvoir être vécu comme ça l'a été ce jour-là, le sein de la première tétée ne devant jamais plus se présenter de la même façon. Das Ding est cet objet perdu pour toujours. Dans tout sujet (au plus intime de lui), il y a donc la marque d'un manque (un lieu vide), le manque de cet objet perdu qui est, en même temps, radicalement étranger à lui. Il s'agit d'un manque central dans le registre du désir, et c'est das Ding, quant à l'Autre absolu du sujet, qu'il est question de retrouver, bien que das Ding soit, par sa nature même, perdu à tout jamais. 
intérieur versus monde extérieur, dès lors qu'il ne s'agit pas d'une extériorité en opposition à une intériorité, mais plutôt de la présence du Réel dans le Symbolique.

Recourant à la topologie, geste très cher à Lacan, je fais appel à une figure: celle du tore, qui peut être entendu comme une espèce de chambre à air, une bouée, par exemple, présentant un orifice central, vide et extérieur. Le fait que ce trou (le vide) soit en même temps au centre et à l'extérieur le rend propice à figurer ce que serait l'«extime».

Mais revenons de nouveau à Budapest. On note que l'objet étranger détecté par Kriska dans le texte écrit (c'est-à-dire, l'«extimité» instaurée par sa parole à elle) est un reste du corps du nègre: un ton de voix qui s'est «détaché» de lui, «se perdant» dans le texte signé par le poète hongrois, qui ne fonctionne plus comme quelque chose de sonore, inducteur de sens, pour provoquer un effet de «trou». Nous observons, par conséquent, que l'espace de l'«extimité» est un espace de la perturbation, ce que l'on peut vérifier encore par ce que dit la jeune femme: «On dirait qu'ils ne sont pas hongrois ces poèmes» (Buarque 2005: 126), ces poèmes-là écrits en hongrois. Il s'agit, en dernier lieu, d'un oxymore.

\section{L'extimité et le corps}

La notion d' «extimité» repérée dans Budapest, en donnant une nouvelle dimension au couple paradigmatique dedans/ dehors, nous permettra à nouveau de noter les diverses références au corps dans l'œuvre de Chico Buarque, avant tout par le biais du couple masculin/féminin ${ }^{18}$.

Comme on a pu le voir au début de ce travail, dans ses premières productions (les chansons des années 60), l'opposition traditionnelle dedans / dehors est maintenue, dans la mesure où un homme met en scène le corps de la femme, qui se situe à part, en dehors de lui. Par exemple, il veut soit apprécier le corps de sa bien aimée ( «Je veux voir la samba chauffer/ dans le corps de la femme porte-étendard / que ma guitare va lever ${ }^{19}$ ), soit

18 Curieusement, l'idée d' «extimité» va jusqu'au domicile de Chico, à Rio de Janeiro, une fois qu'il a appelé «Paris» son bureau, à l'étage inférieur de celui où il vit, mais faisant partie de l'ensemble de son appartement. Un étranger, un dehors, s'installe alors dedans sa maison.

19 «Demain on ne sait pas». 
s'étendre sur son corps («Dans le giron de ma bienvenue compagne / [...] Je fais de la samba et l'amour la nuit entière $\left.»^{20}\right)$.

Dans les années 70 , ce corps féminin se présente parfois tellement «dehors», qu'il arrive à se situer en marge de la société, comme c'est le cas pour la prostituée. Mais ce corps de dehors se retrouve dedans, c'est-à-dire que d'un corps masculin émerge la voix (la parole) féminine. Répétons que, dans ce processus, Chico ne se transforme pas, ne se métamorphose pas comme cela se passe dans le candomblé; lorsqu'il chante, il n'y a pas la moindre simagrée féminine dans ses gestes, ou des modulations dans sa voix de chanteur, capables d'imiter une voix féminine. Il apporte la parole féminine, mais en chantant comme homme; d'ailleurs, un homme que beaucoup de femmes brésiliennes de différents âges jugent extrêmement viril, masculin.

Nous observons donc que, de fait, un renversement s'est opéré dans son processus créatif: de dehors, la parole, la voix féminine, devient dedans. C'est comme si Chico avait préparé quelque chose que lui seul va atteindre dans Budapest: un point optimal de résolution, d'épuration: la voix (non plus spécifiquement féminine, mais la «voix» tout court) sera dedans et dehors en même temps.

Et encore: la notion d' " extimité» repérée dans Budapest nous permet aussi de situer la place de Chico Buarque dans la culture brésilienne contemporaine. Selon nous, le compositeur-écrivain pourrait y occuper un lieu «extime»: intimement impliqué dans les questions politiques de notre pays, Chico se maintient néanmoins en dehors d'elles au niveau institutionnel, dans une relation de méfiance à leur égard. Allant plus loin que ces considérations, je pense aussi que la place «extime» occupée par Chico Buarque dans la contemporanéité refléterait un déplacement en rapport avec la place extraterritoriale occupée par plusieurs artistes et/ou intellectuels dans la modernitée 21.

Dans cette perspective, Budapest se révèle être un pas décisif dans la carrière littéraire de Chico et une œuvre marquante de la littérature

20 «Samba et amour».

21 Un exemple paradigmatique de cette place de l'artiste dans la modernité (d'extraterritorialité et non d' "extimité», comme je considère celle de Chico) peut être donné par Mallarmé. Mettant en valeur le travail de tel artiste, la position de Leyla Perrone-Moisés à ce sujet est celle-ci: "Les tours d'ivoire dans lesquelles s'enfermèrent les poètes de la modernité furent une réaction, nullement une attitude réactionnaire. [...] Mallarmé s'est dissimulé sous la modeste condition de professeur d'anglais pour se dédier, à ses heures creuses, au scandale de sa syntaxe» (Perrone-Moisés 2000: 32-33). 
brésilienne contemporaine. Ce n'est pas un hasard si, à l'occasion de la sortie du livre en français, à Paris, au printemps 2005, le supplément littéraire du journal Le Monde salua l'écrivain comme «l'un des plus intéressants de ceux qui nous sont arrivés du Brésil, ces dernières années» (Rerolle 200522).

\section{Bibliographie}

Bastide, Roger, 2000, Le candomblé de Bahia, Paris, Plon.

Buarque, Chico, 1992, Embrouilles, Paris, Gallimard.

Buarque, Chico, 1997, Court-circuit, Paris, Gallimard.

Buarque, Chico, 2005, Budapest, Paris, Gallimard.

Buarque de Hollanda, Sérgio, 1973, Raízes do Brasil, $7^{e}$ éd., Rio de Janeiro, José Olympio.

Eichemberg, Fernando, 2006: «Chico e suas novas namoradas». http://www.chicobuarque.com.br/texto/index.html.

Lacan, Jacques, 2006, Le Séminaire, Livre XVI, D’un Autre à l'autre (1968-1969), Paris, Seuil.

Lacan, Jacques, 1986, Le Séminaire, Livre VII: L'éthique de la psychanalyse, Paris, Seuil.

Ribeiro, Hamilton, 2006, «Chico põe nossa música na linha». http://www.chicobuarque.com.br/texto/index.html.

Menezes, Adélia Bezerra de, 2001, Figuras do feminino na canção de Chico Buarque, $2^{e}$ éd., São Paulo, Ateliê.

Perrone-Moisés, Leyla, 2000, Inútil poesia, São Paulo, Companhia das Letras.

Rerolle, Raphaëlle, 2005, «Chico Buarque, brésilien du siècle».

http://www.ddooss.org/libros/651548_sup_livres_050519.pdf.

Souza, Eneida Maria de, 2006, "O samba da minha terra», in H.K. Olinto,

H. E. Schollhammer (ss dir.), Literatura e memória, Rio de Janeiro, Galo Branco, p. 45-56.

22 Disponible à : http://www.ddooss.org/libros/651548_sup_livres_050519.pdf. 